\title{
Utilization of visual and tactual cues by the white rat in following a trail'
}

DAVID A. SISEMORE, University of Southern Mississippi, Hattiesburg, Miss. 39401, and HARDY C. WILCOXON, George Peabody College for Teachers, Nashville, Tenn. 37203

White rats learned in approximately seven trials to follow conspicuous visual and tactual cues over varying routes through a modified Dashiell maze. Eight Ss learned to follow a white trail in an otherwise black maze, eight Ss a black sandpaper trail in the same maze, while eight controls practiced the same varying patterns, but with no trail cue. A transfer test, in which Ss that had learned one trail cue were switched to the other, showed that transfer was better from the sandpaper to the white trail than it was vice versa.

Many animals can follow a trail over an erratic course, even though different movements are required at unpredictable times and places to maintain sensory contact with the trail. The hunting dog tracking game is a familiar example. The main interest in the present study was to determine whether the rat is capable of analogous behavior.

Early work on the sensory control of the maze habit in rats occasionally included placing a trail of some kind on the true pathway (Vincent, 1915a, b; Liggett, 1928; Snygg, 1935). These studies, however, all involved giving rats repeated trials on a fixed maze pattern, rather than different ones. Their purpose was to determine how much various cues facilitate ordinary maze learning, rather than to discover whe ther the rat can learn to follow a particular cue over an unpredictable course.

In the present study, rats learned a maze whose pattern of correct turns changed each trial, but whose true pathway was always delineated by a distinctive cue, or trail. Three groups of Ss practiced the same varying maze patterns. One group had a distinctive visual cue marking the true pathway, another a tactual cue, while a control group had no distinctive cue.

\section{SUBJECTS}

The Ss were 24 naive albino rats of the Wistar strain approximately 100 days old at the beginning of the study. They were tamed by daily handling and adapted to a 24-h maintenance schedule for 1 week prior to training. The feeding schedule throughout the study allowed Ss to eat dry lab chow for 30 min after each day's run, with water
Trail Training

Following random assignment of Ss to three groups (visual, tactual, and control), all received one trial per day for 15 days in the main apparatus. A different maze pattern, randomly selected from the 20 possible ones, was used each day for all groups. For the visual group the floor of the true pathway was covered with flat white plywood squares, while flat black pieces covered the floor of all blinds. Thus, the true pathway was indicated by a continuous white trail from start to goal. The tactual group had a similar trail made of sandpaper-covered plywood painted the same flat black as that in the blinds. The control group had no trail; flat black plywood was in the true pathway as well as the blinds.

\section{Transfer to Different Cue}

For the next 5 days, the remaining five maze patterns were used in a transfer test. The visual group was switched to the tactual trail condition and the tactual group to the visual. Control group Ss were randomly assigned in equal numbers to the visual and tactual trail conditions to determine whether they would follow a novel stimulus trail upon first encountering it. As above, all Ss received one trial per day on a maze pattern which was different each day.

Throughout the study the maze was rotated at random each day to one of the four cardinal compass positions, the identical goal and starting boxes were arbitrarily redesignated daily, and the order of running Ss was counterbalanced within and between groups.

The experiment was run in two replications of four Ss per group. Running time and errors per trial were recorded. An error was recorded when $S$ touched, so as to move it, any curtain other than one leading toward the goal.

\section{RESULTS}

Trail Learning

After Ss were adapted to the feeding schedule, they were trained for 7 days to run down a 6-ft runway to moist lab chow. Curtains like those in the main apparatus were placed in the runway during the last 4 days.

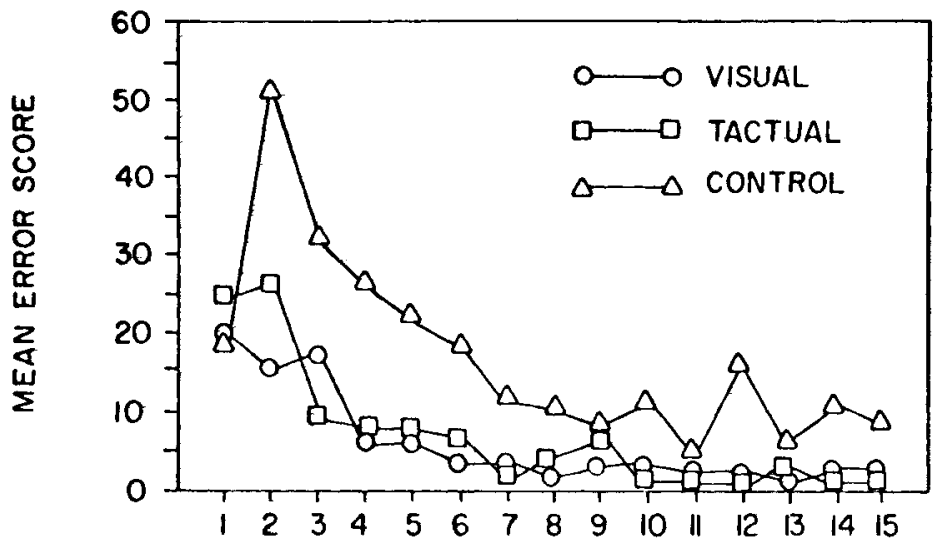

MAZE PATTERN 


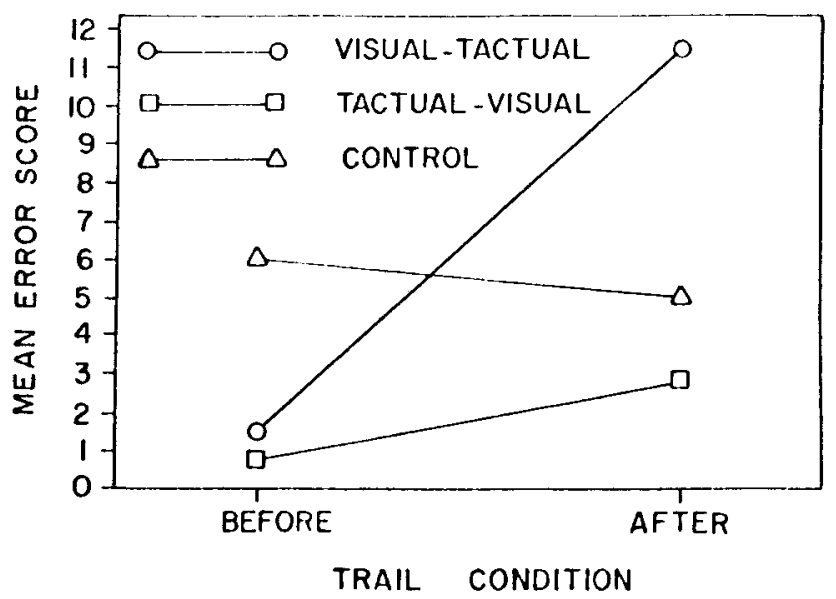

Fig. 2. Mean errors on the last trial before the change in trail condition, and the first trial after the change in trail condition.

Running time closely paralleled errors throughout and will not be reported further.

Both the visual and the tactual groups showed a much greater reduction in errors than the control group, as seen in Fig. 1, although the control group itself performed better, also, as practice continued. [Control Ss were probably learning what Dashiell (1930) called "directional orientation," i.e., a general forward-going tendency from the start box, with fewer retracing and perseverative errors.]

Sheffé's test (Sheffé, 1953), applied to the mean differences in errors made by the three groups, showed that each trail-cue group made significantly fewer errors than the control group $(p<.01)$, and that the visual and tactual groups were not significantly different from each other $(\mathrm{p}>.05)$.

Further evidence that $S$ s in the visual and tactual groups were following the trails came from looking at the raw scores. Three of the experimental Ss made no errors on five or more successive trials, and 15 of the 16 succeeded in running at least one maze pattern errorlessly. In contrast, only one of the eight control Ss ran as much as one errorless trial.

\section{Transfer to Different Cue}

As seen in Fig. 2, the tactual group showed a high degree of transfer to the visual trail on the first trial following the change in cue. The slight increase in errors accompanying the cue change was not statistically significant. The visual group, on the other hand, showed a large increase in errors $(p<.01)$ on the first trial using the tactual trail. The performance of the control group remained approximately the same $(p>.05)$.

Error data over all five trials following the change in trail cues were also analyzed. This analysis showed that tactual-to-visual Ss made fewer errors than either of the other two groups $(p<.01)$. The difference, however, between the visual-to-tactual group and the control group, which now had a trail to follow, was nonsignificant $(p>.05)$.

\section{DISCUSSION}

That white rats can easily learn to follow a visual or tactual trail has interesting theoretical implications. The rapidity of the learning, especially, does not appear to be readily predictable from the major, traditional explanations of maze learning. No fixed s-r sequences that would lead to errorless performance could have been formed in this situation, since a different pattern of turns was required each trial. For the same reason, an accurate cognitive map could not have been acquired. Instead, Ss seem to have had to learn to pay attention (Lawrence, 1949 , 1950 ) to a particular cue, and then to follow it wherever it led. The results suggest, therefore, that a central, attentional process is involved, even in this relatively simple situation.

Regardless of theoretical interpretation the ease with which Ss in both experimental groups learned to follow trails was not expected; many were performing errorlessly by the seventh trial. A probable reason for the rapid learning, however, was the shortness of the blinds, which made the consequences of responding almost immediate, and which probably favored quick development of attention to the relevant cues. When $S$ made an error, his nose quickly came into contact with the hard surface of a block, and this occurred almost contiguously with a distinct change in the floor surface. Similarly, when a correct choice was made, $S$ was immediately free to push under a curtain and continue running toward the goal on a homogeneous surface. Such conditions of immediate feedback from responses made in the presence of relevant cues have been shown to favor rapid learning in many other situations, notably Jarvik (1953) and Wodinsky, Varley, \& Bitterman (1954).

Several factors might account for superior transfer from the tactual to the visual trail than vice versa. The following suggestions are quite tentative, therefore.
First, the tactual group might actually have learned to follow the original trails visually, rather than tactually. The sandpaper trails were clearly visible to $E$, and probably to Ss, for despite use of the same flat black paint on the trails and the plywood flooring of the blinds, the trails were slightly lighter in color.

Assuming, then, that $S$ s in both experimental groups were responding primarily to visual cues both before and after the change in trail cues, the transfer results can be accounted for as follows. The change from the tactual to the visual trail condition was, for S, simply a change from a weak to a strong visual stimulus. On the other hand, the change from the visual to the tactual trail condition amounted to a change from a strong to a weak visual stimulus. Since generalization is usually greater from weak to strong stimuli than it is from strong to weak (Kimble, 1961, p. 342), better transfer would be expected in the tactual-to-visual group than in the visual-to-tactual group.

In conclusion, the experiment demonstrates that white rats can quickly learn to track, in the cybernetic sense, a distinctive stimulus trail that leads to food over an unpredictable course in a maze. We suggest that an appropriate model for such behavior should include a central, attentional process, for it appears that $S$ quickly learns to pay attention to the relevant cue, and is then able to follow it over whatever erratic course it takes. Of the trail cues used in the present study, the visual trail appeared to be the more perspicuous cue, especially in the transfer test.

\section{REFERENCES}

DASHIELL, J. F. Directional orientation in maze running by the white rat. Comparative Psychology Monographs, 1930, 7 (Whole No. 32).

JARVIK, M. E. Discrimination of colored food and food signs by primates. Journal of Comparative \& Physiological Psychology, 1953, 46, 390-392.

KIMBLE, G. A. Hilgard and Marquis' conditioning and learning. (Rev. ed.) New York: Apple tonCentury-Crofts, 1961.

LAWRENCE, D. H. Acquired distinctiveness of cues: I. Transfer between discriminations on the basis of familiarity with the stimulus. Journal of Experimental Psychology, 1949, 39, 770-784.

LAWRENCE, D. H. Acquired distinctiveness of cues: II. Selective association in a constant stimulus situation. Journal of Experimental Psychology, 1950, 40, 175-188.

LIGGETT, J. R. An experimental study of the olfactory sensitivity of the of the white rat. Genetic Psychology Monographs, 1928, 3 (Whole No. 1).

SCHEFFÉ, H. A method for judging all contrasts in the contrasts in the analysis of variance. Biometrika, 1953, 40, 87-104.

SNYGG, $D$. The relative difficulty of mechanically equivalent tasks. II. Animal learning. Journal of Genetic Psychology, 1935, 47, 321-336.

VINCENT, S. B. The white rat and the maze problem. I. The introduction of a visual control. Journal of Animal Behavior, 1915a, 5, 1-24. 
VINCENT, S. B. The white rat and the maze problem. II. The introduction of an olfactory control. Joumal of Animal Behavior, 1915b, 5 , $140-157$.

WODINSKY, J., VARLEY, M. A., \& BITTERMAN, M. E. Situational determinant of the relative difficulty of simultaneous and successive discrimination. Journal of Compara- tive \& Physiological Psychology, 1954, 47, 337-340.

\section{NOTE}

1. Based on a thesis submitted to the University of Arkansas by the first author in partial fulfillment of the requirements of the Master of Arts degree.

\section{Schedule-induced polydipsia: Effects of providing an alternate reinforced response and of introducing a lick-contingent delay in food delivery'}

\begin{abstract}
EVALYN F. SEGAL, University of Illinois at Chicago Circle, Chicago, Ill. 60680 and DAVID L. ODEN, University of Pennsylvania, Philadelphia, Pa. 19104
\end{abstract}

In Experiment 1, rats exhibited polydipsia on a concurrent spaced-response, variable-ratio schedule of reinforcement for bar presses on two bars, indicating that even the availability of a reinforcement schedule $(V R)$ that encourages high and steady bar-pressing rates does not eliminate the polydipsia induced by the spacing of food. In Experiment 2, rats developed polydipsia in response to a spaced-food schedule, even though every water lick delayed food delivery for 30-60 sec, indicating that the development of polydipsia does not depend on adventitious food reinforcement.

\section{EXPERIMENT}

Earlier studies have indicated that polydipsia will occur whenever fooddeprived $S s$ are exposed to a food-delivery schedule that delivers a food pellet at a spacing greater than about $20 \mathrm{sec}$, whether or not food deliveries are contingent on the emission of an operant response (Burks, Hitzing, \& Schaeffer, 1967; Clark, 1961; Deadwyler \& Segal, 1965; Falk, 1961a, b, 1964, 1966a, b, c, 1967, in press; Kaplan, 1965; Lester, 1961; Malott \& Cumming, 1965; Salzberg, 1967; Salzberg\& Schaeffer, 1968; Schaeffer \& Diehl, 1966; Schaeffer, Diehl, \& Salzberg, 1966; Schuster \& Woods,
1967; Segal \& Bandt, 1966; Segal \& Holloway, 1963; Segal, Oden,\& Deadwyler, 1965; Stein, 1964). Schedules that provide food deliveries very close together, however, do not usually elicit polydipsia. Small fixed-ratio, fixed-interval, or variableinterval bar-pressing schedules do not cause polydipsia, nor does a continuous reinforcement schedule, nor do free-food schedules that deliver food as often as every $20 \mathrm{sec}$. The fact that polydipsia occurs only when the spacing of food deliveries reaches some minimum time interval suggests that the drinking might partly be accounted for by the animal's impatiently filling time with some behavior that makes waiting for the next food delivery tolerable. This suggestion is weakened by the fact that rats will exhibit polydipsia on schedules, such as a variableinterval bar-press reinforcement schedule, that might be thought to encourage the rat to fill time by bar pressing. It is weakened further by the finding (Segal, in press) that polydipsia will occur even when an alternate response (wheel running) is available to the animal to fill time with. Finally, the present data weaken the time-filling hypothesis still further by showing that polydipsia occurs even when a reinforced alternate response (bar pressing on a variable-ratio schedule) is available, and even when the reinforcement schedule is one that encourages high and steady rates of bar pressing-which might be thought to leave no empty time to be filled with drinking.

\section{Method}

One male, young adult, albino rat was maintained at $80 \%$ of ad lib weight by a food-deprivation regimen, and exposed for several sessions to a concurrent DRL 20-sec VR 23 bar-pressing schedule on two levers. Presses on one lever were reinforced if they were spaced at least $20 \mathrm{sec}$ from the preceding press on that lever (DRL $20 \mathrm{sec}$ ); presses on the other lever were reinforced after a variable number of presses, averaging 23 , was emitted. The reinforcers were 45-mg Noyes rat chow pellets, and sessions lasted until 150 reinforcers had been received. A water bottle was available.

Results

Figure 1 shows typical results from Session 11. Presses on the DRL bar occurred at the low, steady rate characteristic of performance on spaced-response DRL schedules. Presses on the VR bar occurred at the high and fairly steady rate characteristic of performance on variable-ratio schedules. Nevertheless, a polydipsic level of drinking occurred. The drinking was not so persistent, however, as on a simple DRL 20-sec schedule (e.g., see the figure in Segal

\section{RAT 5 - DRL 20 VR 23 - DAY \|}
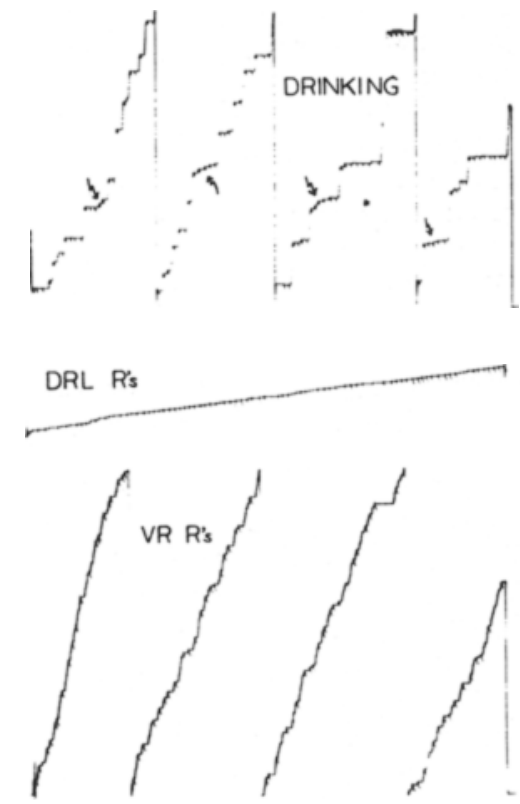

Fig. 1. Cumulative licks (upper tracing), cumulative bar presses on the DRL (spaced response) bar (middle tracing), and cumulative bar presses on the VR (variable ratio) bar (lower tracing), in Session 11 of concurrent DRL 20-sec VR 23. Each full excursion of the pen represents about 500 responses. Downward deflections in the drinking record indicate DRL bar presses; in the other two records they indicate reinforcements on the respective schedules. 